
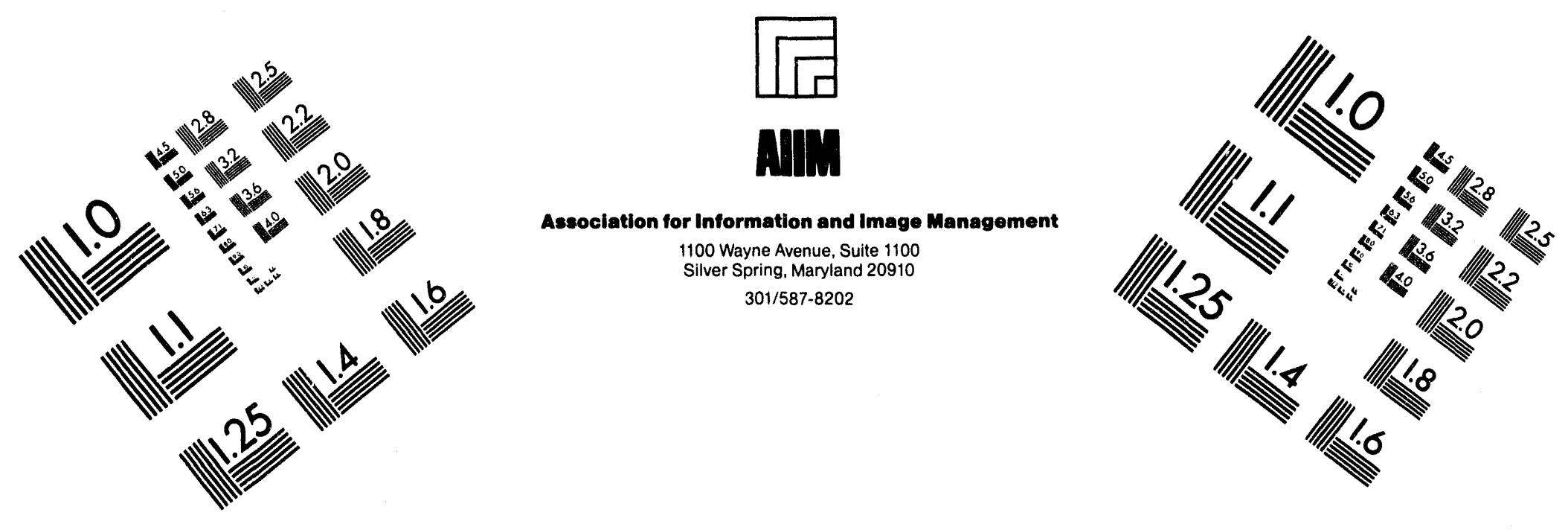

Centimeter

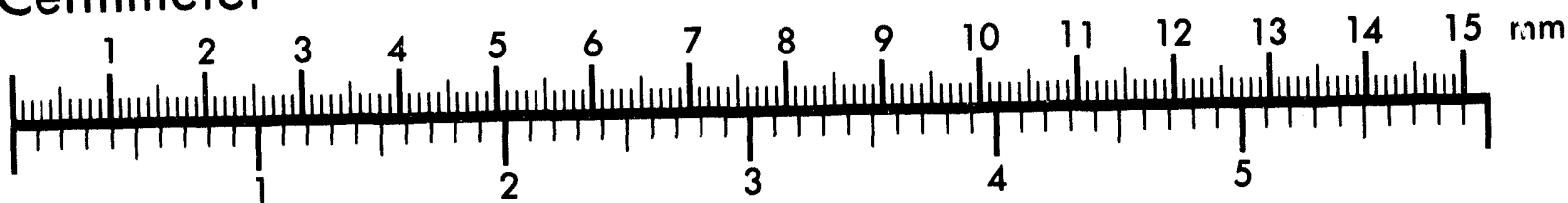
Inches
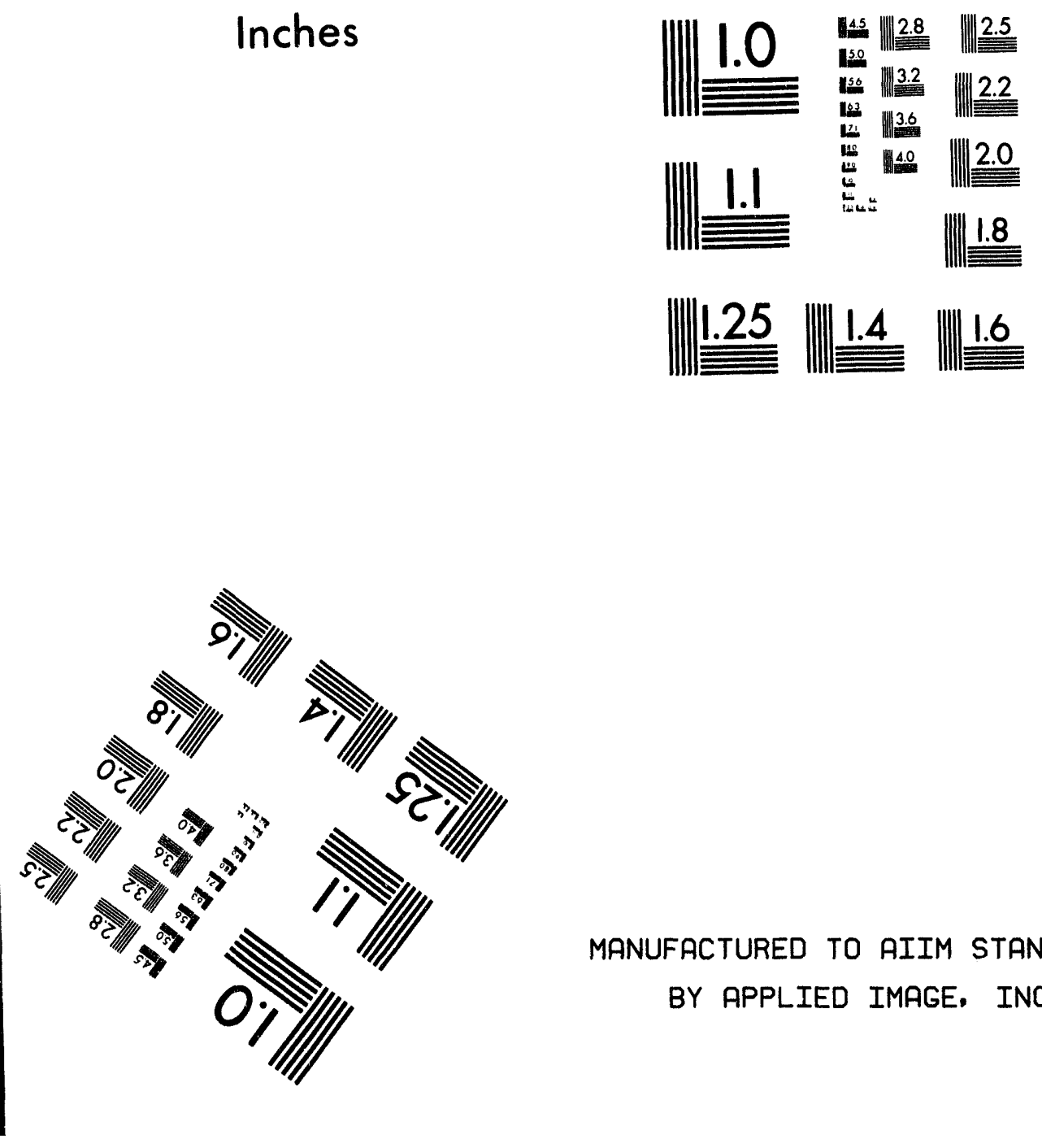

MANUFACTURED TO AIIM STANDARDS BY APPLIED IMAGE, INC.

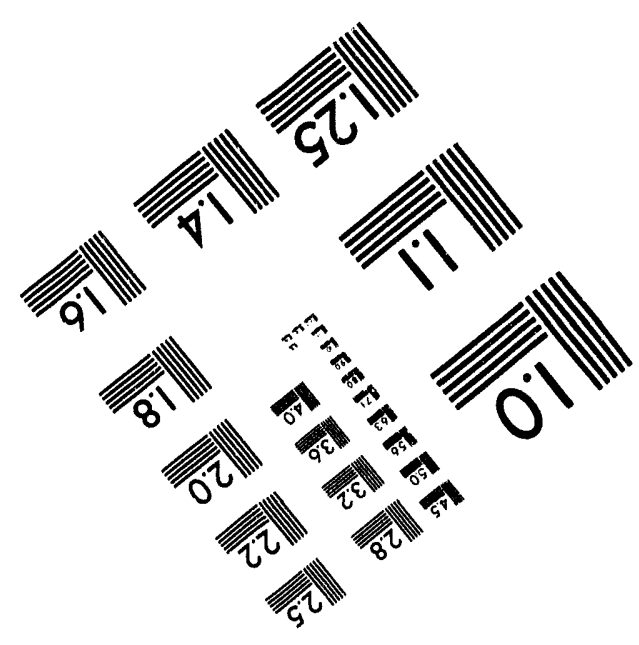





\section{Conf-940391-..7}

UCRL-JC-114939

PREPRINT

An adaptive optics package designed for astronomical use with a laser guide star tuned to an absorption line of atomic sodium

\section{J. Thaddeus Salmon, Ken Avicola, James M. Brase, John W. Bergum, Herbert W. Friedman, Donald T. Gavel, Claire E. Max, Stephen D. Mostek, S. S. Olivier, Robert W. Presta, Rodney J. Rinnert, Charles W. Swift, Kenneth E. Waltjen, \\ Carolyn L. Weinzapfel and J. Nan Wong}

This paper was prepared for submittal to SPIE's 1994 Symposium on Astronomical Telescopes \& Instrumentation for the 21st Century Kona, Hawaii March 13-18, 1994

April 11, 1994

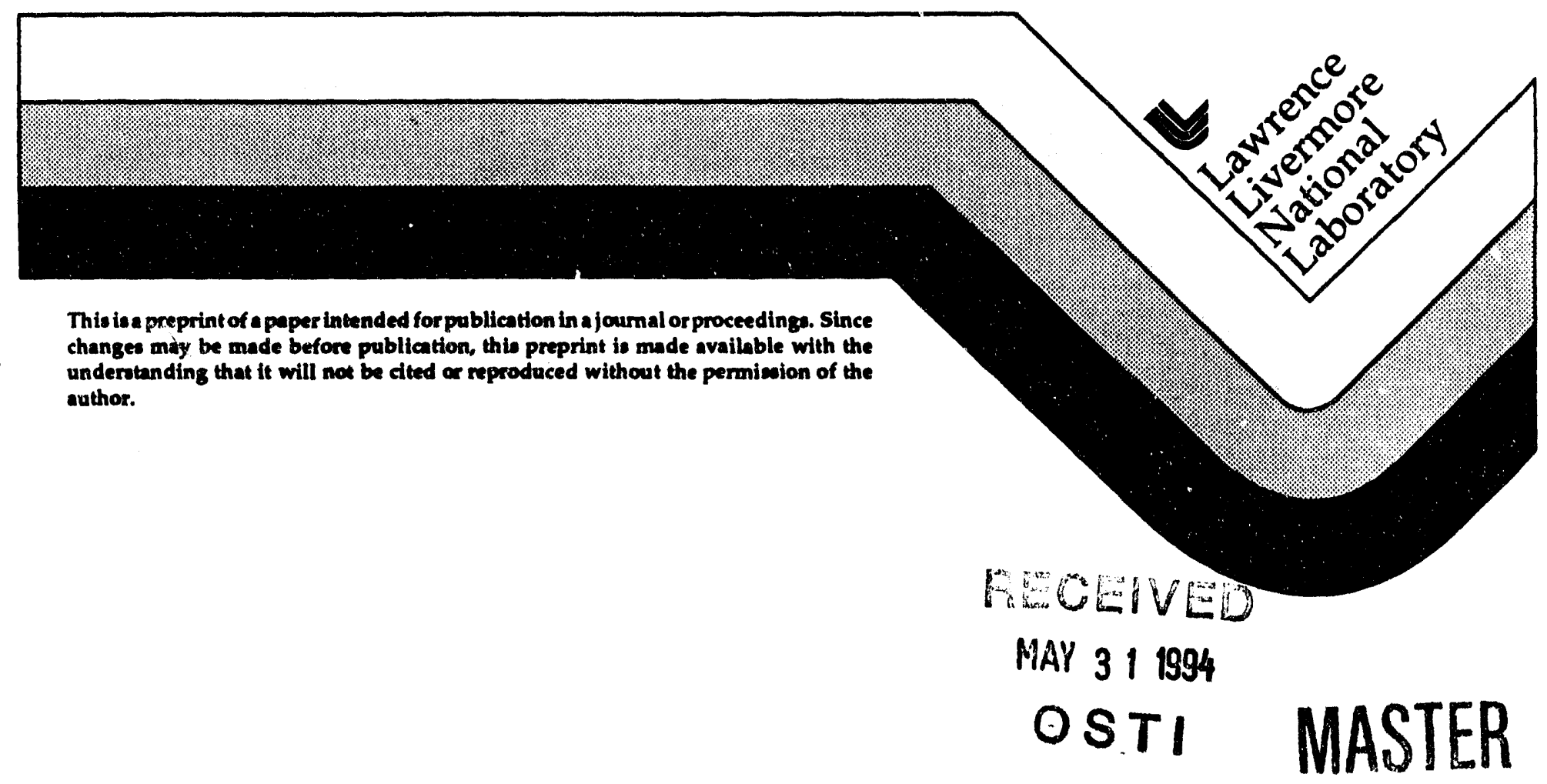


This document was prepared as an account of work sponsored by an agency of the United States Government. Neither the United States Government nor the University of California nor any of their employees, makes any warranty, express or implied, or assumes any legal liability or responsibility for the accuracy completeness, or usefulness of any information, apparatus, product, or process disclosed, or represents that its use would not infringe privately owned rights. Reference herein to any specific commercial product, process, or service by trade name, trademark, manufacturer, or otherwise, does not necesearlly constitute or imply its endorsement, recommendation, or favoring by the United States Government or the University of California. The views and opinions of authors expressed herein do not necessarily state or reflect those of the United States Government or the University of California, and shall not be used for advertising or product endorsement purposes. 


\title{
An adaptive optics package designed for astronomical use with a laser guide star tuned to an'absorption line of atomic sodium
}

J. Thaddeus Salmon, Ken Avicola, James M. Brase, John W. Bergum, Herbert W. Friedman, Donald T. Gavel, Ciaire E. Max, Stephen D. Mostek, S. S. Olivier, Roben W. Presta, Rodney J. Rinnert, Charles W. Swift, Kenneth E. Waltjen, Carolyn L. Weinzapfel, J. Nan Wong

Lawrence Livermore National Laboratory, Livermore, CA 94550

\begin{abstract}
We present the design and implementation of a very compact adaptive optics system that senses the return light from a sodium guide-star and controls a deformable mirror and a pointing mirror to compensate atmospheric perturbations in the wavefront. The deformable mirror has 19 electrostrictive actuators and triangular subapertures. The wavefront sensor is a Hartmann sensor with lenslets on triangular centers. The high-bandwidth steering mirror assembly incorporates an analog controller that samples the tilt with an avalanche photodiode quad cell. An $f / 25$ imaging leg focuses the light into a science camera that can either obtain long-exposure images or speckle data. In laboratory tests overall Strehl ratios were improved by a factor of 3 when a mylar sheet was used as an aberrator. The crossover frequency at unity gain is $30 \mathrm{~Hz}$.
\end{abstract}

\section{INTRODUCTION}

The concept of using the mesospheric sodium layer at an altitude of about $90 \mathrm{~km}$, excited with a resonantly tuned laser as a means of forming a point reference, or guide star, has been under investigation for several years. ${ }^{1-5}$ The laser guide star program at LLNL started in 1990 and is a Laboratory funded research and development program. The goal has been to demonstrate the feasibility of closed-loop adaptive optics with sodium laser guide stars. A high average power tunable dye laser system, then in routine operation at LLNL, provided the laser source for these experiments. This laser system was developed for the Department of Energy's Atomic Vapor Laser Isotope Separation (AVLIS) program, ${ }^{6}$ and could easily be tuned to the required $589 \mathrm{~nm}$ wavelength and the spectral width broadened to $3 \mathrm{Ghz}$.

The adaptive optics package described in this paper was designed for laser guide star experiments using the AVLIS laser as the source and a half-meter telescope to observe the sodium spot in the mesosphere. The laser system and initial photometric experiments on the laser guide star are described by Avicola et al. in a companion paper at this conference. ${ }^{7}$ In October 1993 the laser was shut down and placed in cold standby until the future course of AVLIS is decided. Hence, experiments planned for the atmosphere and a sodium guide star were moved into the laboratory with a simulated source and an aberrator that approximated the atmosphere. The results of these laboratory experiments are presented here.

\section{SYSTEM DESCRIPTION}

The adaptive optics package, shown in Fig. 1, is designed to fit at the Cassegrain tivils of a half-meter telescope and operates at wavelengths ranging from $700-900 \mathrm{~nm}$. The package, which is very compact, is assembled on an optical breadboard that is $0.75 \mathrm{~m} \times 1.22 \mathrm{~m}$, and the light enters through a port in the breadboard that is to the left of center in the figure. The light immediately enters and focuses within a reverse Cassegrain assembly, which recollimates the light and relays the input pupil of the telescope, the primary mirror, onto the deformable mirror. The design places the high-bandwidth tilt mirror immediately before the deformable mirror, since placing the tilt mirror between the deformable mirror and the wavefront sensor would shift the image of the deformable mirror on the lenslet array of the Hartmann sensor when the tilt loop is closed. This shift would adversely affect the stability of the high-order control of the wavefront. Figure 2 is a photograph of the adaptive optics package assembled and tested. The front comer of the breadboard corresponds to the lower right comer of the

"Work performed under the auspices of the U.S. Department of Energy by the Lawrence Livermore National Laboratory under Contract W-7405-Eng-48. 
layout in Fig. 1. The deformable mirror is just to the left of center, and the Hartmann sensor is at the rear comer of the breadboard. The high-bandwidth detector is above and behind the right side of the scale at the front of the breadboard.

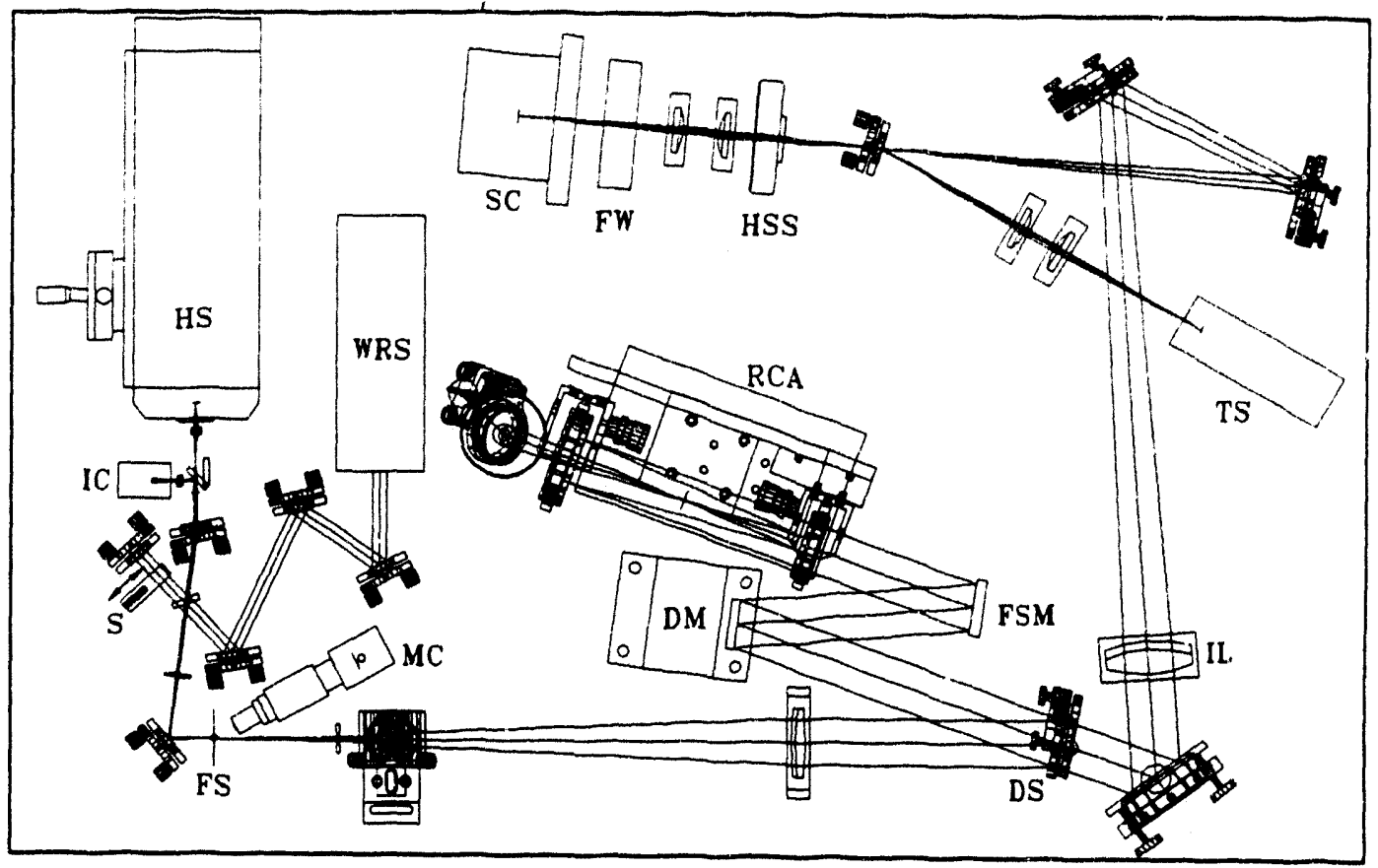

Fig. 1 Schematic of the layout of the adaptive optics package showing the deformable mirror (DM), the field stop (FS), the fast steering mirror (FSM), the filter wheel (FW), the Hartmann sensor (HS) and associated relay telescope, the high-speed shutter (HSS), the imaging camera (IC), the imaging lens (IL), the microscope camera (MC), the collimating Reverse Cassegrain assembly (RCA), shutter (S), the science camera (SC), the tilt sensor (TS), and the wavefront reference source (WRS).

The adaptive optics system is based on a system designed for AVLIS and described by Salmon et al. ${ }^{8}$ This system uses a deformable mirror that has triangular subapertures and a Hartmann sensor that has lenslets with centers on a triangular grid. In contrast to adaptive optics systems that use Hartmann sensors and deformable mirrors with square subapertures, this system does not suffer from any modes of the deformable mirror that are not detectable by the Hartmann sensor. The undetected mode arises from the reconstructor for a Hartmann sensor with lenslets on a rectilincar grid having two independent sets of equations with the configuration of the two sets conforming to white and black squares of a chess board and is described by Fried. ${ }^{9}$ The alignment of the lenslets of the Hartmann sensor to the actuators of the deformable mirror is shown in Fig. 3. The redundancy in lenslets with respect to actuators renders the reconstructor less sensitive to noise in the measurements by the Hartmann sensor. Also shown are the clear aperture, $42 \mathrm{~mm}$, and the obscuration by the secondary mirror of the telescope. The lenslets of the Hartmann sensor are superimposed on the mirror and show the alignment of the Harmann sensor to the actuators of the mirror that provides the optimal control of the reflected wavefront.

The deformable mirror, shown in Fig. 4, uses 19 electrostrictive actuators of lead magnesium niobate (PMN). The actuators have a nominal stroke of $8 \mu \mathrm{m}$ at 80 volts and have about $3-4 \%$ hysteresis. The size of the mirror is $45 \mathrm{~mm}$ side-to-side with $11.5 \mathrm{~mm}$ between actuators. This size of subaperture on the deformable mirror translates to an effective subaperture of $14 \mathrm{~cm}$ at the primary mirror of the half-meter telescope. The stroke efficiency of the mirror-actuator force train yields about one wave of change in the reflected wavefront at a wavelength of $633 \mathrm{~nm}$ for each $\mu \mathrm{m}$ of stroke of an unloaded actuator. The influence function of the mirror yields about $1 / 3$ of the deformation of the mirror surface over the neighboring actuators compared to the deformation of the surface above the moved actuators. The actuators outside of the clear aperture are actively 
controlled by lenslets that lie within the clear aperture-there is no need for a guard ring of actuators "slaved" to actuators that lie within the clear aperture. The range in reflected wavefront of the deformable mirror, \pm 10 waves $p-v$, enables us to compensate automatically for focus errors introduced by thermal contraction of the optomechanical structure as the night progresses. The fabricated assembly in closed-100p control has a residual wavefront error in the clear aperture of 0.04 waves rms, which yields a Strehl ratio of 0.94 . Removing the central 7 lenslets from the control, which simulates the obscuration by the secondary of the telescope, only increases the residual wavefront error to 0.05 waves ms. The mirror is designed for easy removal of an actuator that fails with turnaround times (1-2 days) limited to the time is takes to cure the adhesive. ${ }^{10}$ At this time we have not lost any actuators under normal operation. The triangular arrangement of actuators naturally yields a hexagonal mirror shape that closely approximates a circular aperture and is easily scalable to telescopes such as the Keck telescope, which is an array of hexagonal mirrors.

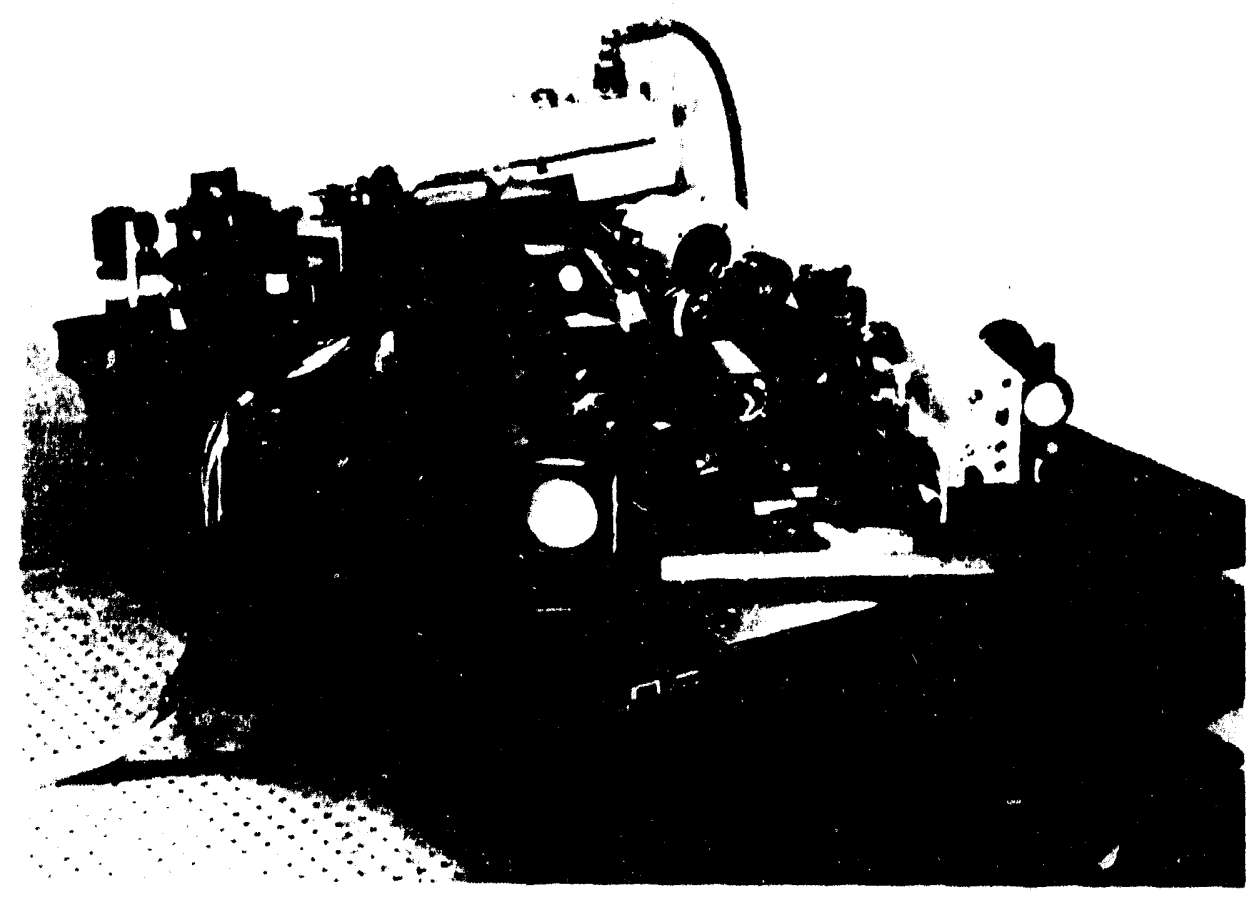

Fig. 2 Photograph of the adaptive optics package.

The Hartmann sensor uses a monolithic microlens array fabricated by Coming. The focal length is set by sandwiching fluid with the appropriate index of refraction between the lenslet array and an optically flat window. 11 The focal length is determined so that the diameter of the Hartmann spots in the focal plane is half of the distance between lenslets in the array. This setting yields the greatest sensitivity while maintaining the range needed for monitoring the wavefront when the system is in open-loop operation. A single lens after the lenslet array reimages the focal plane of the lenslets to the photocathode of the image intensifier on the high-speed camera. The size of the array of Hartmann spots is varied by adjusting the distances between the reimaging lens and both the lenslet array and the photocathode. The detector in the Hartmann sensor is a Kodak EktaPro 1012 intensified high-speed video camera, which is part of a commercial motion analysis system. The electronic interface between the Kodak camera and the controller is a custom board that translates the nonstandard order that the pixel data leave the camera to a form that is amenab!s to the controller.

An insertable mirror and another imaging lens relay the plane of the lenslet array to another CCD detector. This detector is used with a wire mask that can be inserted immediately in front of the deformable mirror. By imaging the wire mask on this lenslet image detector, the afocal telescope in the wavefront leg of the package can be adjusted to set image relay, magnification, rotation, and lateral alignment of the image of the deformable mirror with respect to the lenslet array on the Hartmann sensor. 


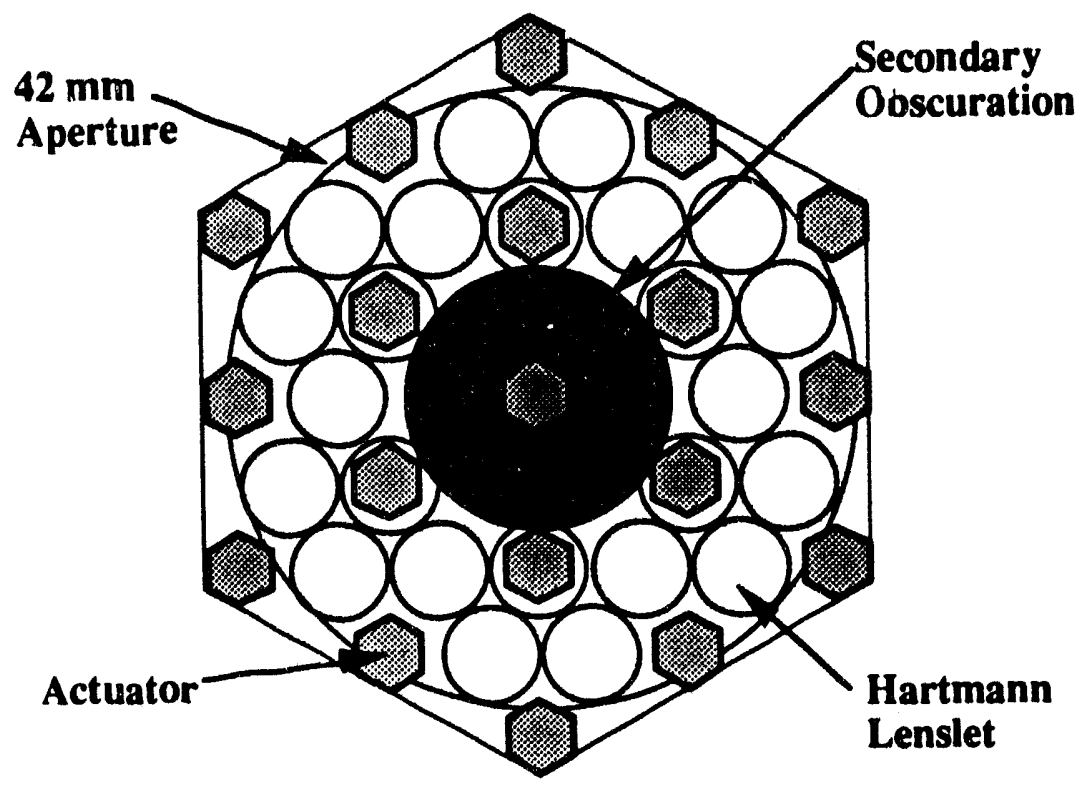

Fig. 3 Schematic of the deformable mirror substrate showing the triangular arrangement of actuators on the back, along with the circular $42 \mathrm{~mm}$ clear aperture used here. Lenslets of the Hartmann sensor are also superimposed to show the alignment of that sensor to the deformable mirror actuators.

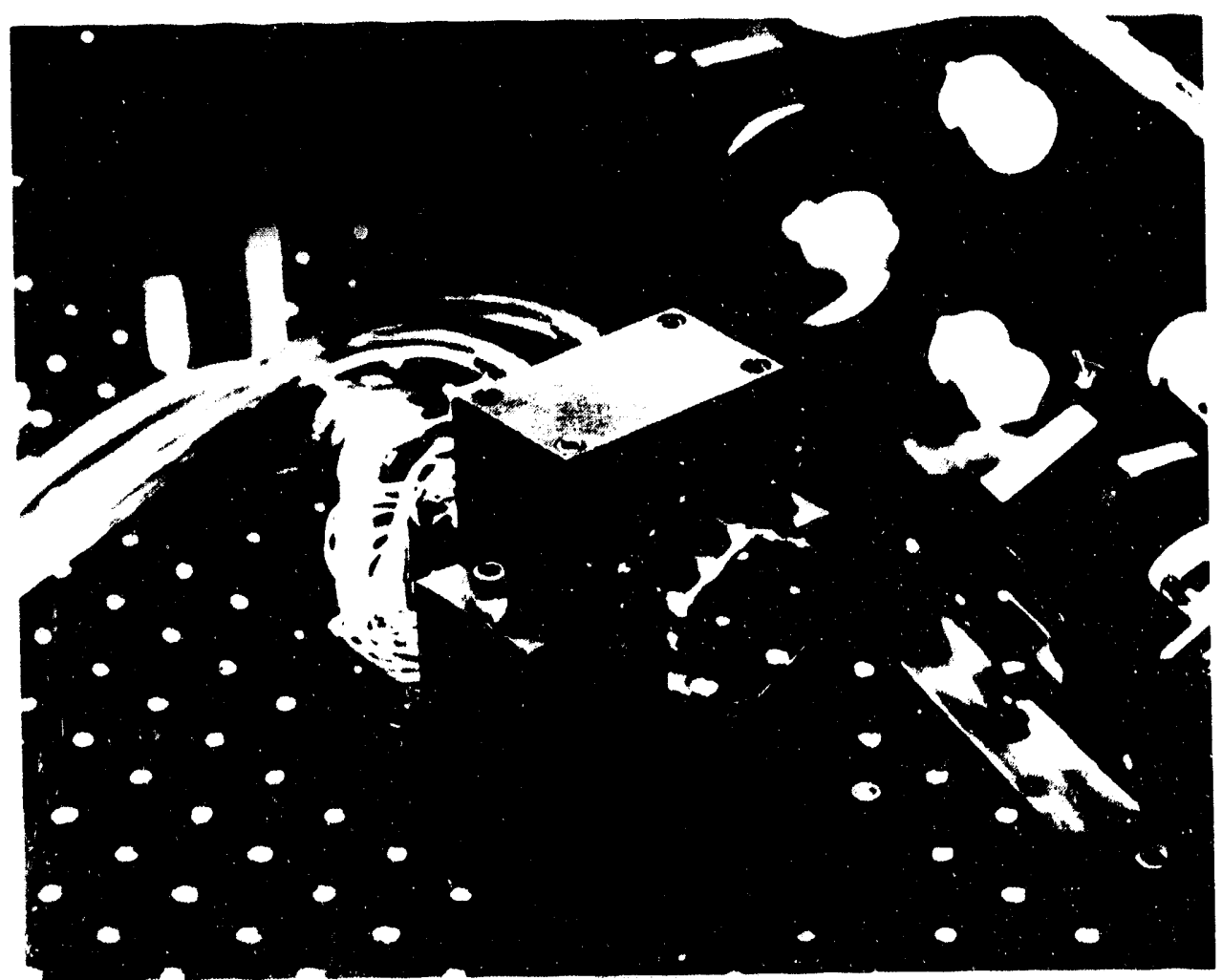

Fig. 4 Photograph of the 19-actuator deformable mirror used in the adaptive optics package. 
The adaptive optics controller is a Mercury quad i-860 array processor VME card that has a high-speed parallel digital interface to the Kodak camera. The details of the controller can be found in Brase et al. ${ }^{12}$ The calculation of the centroids of the Hartmann spots, the reconstruction of the wavefront, and the generation of the control signals are each performed in parallel across three i-860s. The fourth i-860 controls the overall process and provides the diagnostics. The control values are written to digital/analog converters that are connected directly to the deformable mirror drivers via the P2 connection in the VME chassis. This configuration minimizes the number of connectors in the system and, hence, maximizes the reliability of the hardware. The drivers are designed and fabricated in house, and each driver is capable of supplying enougin current to drive a $6 \mu \mathrm{f}$ load at $150 \mathrm{~V}$ peak-10-peak at $1 \mathrm{kHz}$.

The fast steering mirror assembly is described in detail by Avicola et al. ${ }^{7}$ This assembly uses a small flat mirror carefully bonded to a tilt stage by Physik Instrumente to prevent deformation of the front surface. The detector is a quad cell of avalanche photodiodes that is in a plane conjugate to the image plane of the package. The output from the quad cell is processed in an analog controller that uses bi-quadratic control. The unity gain crossover can be set at $30 \mathrm{~Hz}, 60 \mathrm{~Hz}$, or $120 \mathrm{~Hz}$, depending on the seeing conditions.

\section{SYSTEM PERFORMANCE}

The package was assembled in the laboratory with the breadboard about $30 \mathrm{~cm}$ above an optical table. The output from a HeNe laser operating at a wavelength of $594 \mathrm{~nm}$ was expanded and collimated to provide a $36 \mathrm{~mm}$ diameter heam. The beam was then focused with an $\mathrm{f} / 8$ lens that was apertured with an iris in a plane conjugate to the plane of the deformable mirror. The aberration of the light by the atmosphere was simulated with a sheet of mylar that rotated at about 3 revolutions per second. During these tests the output from the Kodak camera was sent to both the controller and the dual-ported video memory in the motion analysis system to record the measured wavefront error. The results presented here are derived from both the stored output from the Kodak camera and the output from a CID video microscope in the focal plane of the package, which corresponds to the image plane of the telescope where a scientific camera or other instrument would be mounted.

The system can independently close the tilt loop and the adaptive optics loop. The frame rate of the Hartmann sensor was $1 \mathrm{kHz}$ and the proportional gain was 0.5. The science camera was replaced with a linear CID camera for these experiments, and a microscope objective magnified the focal plane of the science leg of the package. The plate scale in this camera is equivalent to 0.087 arc sec per pixel when the package is mounted on the $1 / 2$ meter telescope. The diffraction limited spot on this detector is $15.4 \mu \mathrm{m}$ (fwhm), and the effective pixel size, accounting for the microscope objective, is $5.6 \mu \mathrm{m}$. Hence, the diffraction limited spot size is about 3 pixels (fwhm). The output from the Hartmann sensor was stored in the dual ported video memory, as well as used by the controller. This data enabled us to measure directly how well the controller corrected the aberrator.

The light source was a HeNe laser operating at a wavelength of $594 \mathrm{~nm}$. The light from the laser was attenuated, expanded to a diameter of $100 \mathrm{~mm}$, and recollimated. The simulated aberration source was a transparent sheet of mylar on a rotation stage rotating at about $3 \mathrm{~Hz}$. Just before entering the adaptive optics package, the light was apertured with an iris and focused with a lens. The iris was in a plane conjugate to the deformable mirror and simulated the aperture of the primary mirror of the telescope; the focal ratio of the converging beam was 8.2, which matches that of the half-meter telescope.

The controller processed and stored 1024 frames captured by the Harmann sensor during both open loop and closed loop operation. The results are shown in Fig. 5, which shows the $x$-component and the y-component of the power spectrum of the local gradient of the wavefront averaged over all lenslets of the Hartmann sensor. The power spectrum was filtered in the time domain with a low-pass Gaussian filter with a $1 / \mathrm{e}^{2}$ point of $200 \mathrm{msec}$, which removed a sharp resonance in the autocorrelation function at about $330 \mathrm{msec}$. This resonarce corresponds to the $3 \mathrm{~Hz}$ frequency of the rotating mylar sheet. Also shown is the disturbance rejection for the two components of the gradient.

The unity gain crossover is at about $30 \mathrm{~Hz}$ with $7-10 \mathrm{~dB}$ of rejection at the low frequencies; however, the overshoot in the high frequencies results in a gain as high as 3 at $100 \mathrm{~Hz}$. The slope of the open loop power spectra shows that the spectral power of the disturbance is proportional to the frequency $\mathrm{f}^{-4 / 3}$, compared to $\mathrm{f}^{-8 / 3}$ for Kolmogorov urbulence..$^{13}$ Hence, compared to Kolmogorov turbulence, the simulated aberrator has more power at the higher frequencies and puts higher demands on the adaptive optics system. The power spectrum of the reconstructed wavefront error also shows a proportionality to $f^{-4 / 3}$ with residual tilt included and shows a proportionality to $f^{5 / 3}$ with tilt removed. Using the 
reconstructed wavefront errors with tilt removed, the unity gain crossover is at about $50 \mathrm{~Hz}$ with $20 \mathrm{~dB}$ of rejection at the low frequencies, while the overshoot gain is 5 at about $100 \mathrm{~Hz}$. There is also a sharp spike in the disturbance rejection at $90 \mathrm{~Hz}$. This spike also appears on disturbance rejection plots for the reconstructed phase error, both with tilt included and with tilt removed. The appearance of the spike in the spectrum with tilt removed suggests that the source is not vibration of a mirror mount and that the spike is an artifact of the adaptive optics controller.

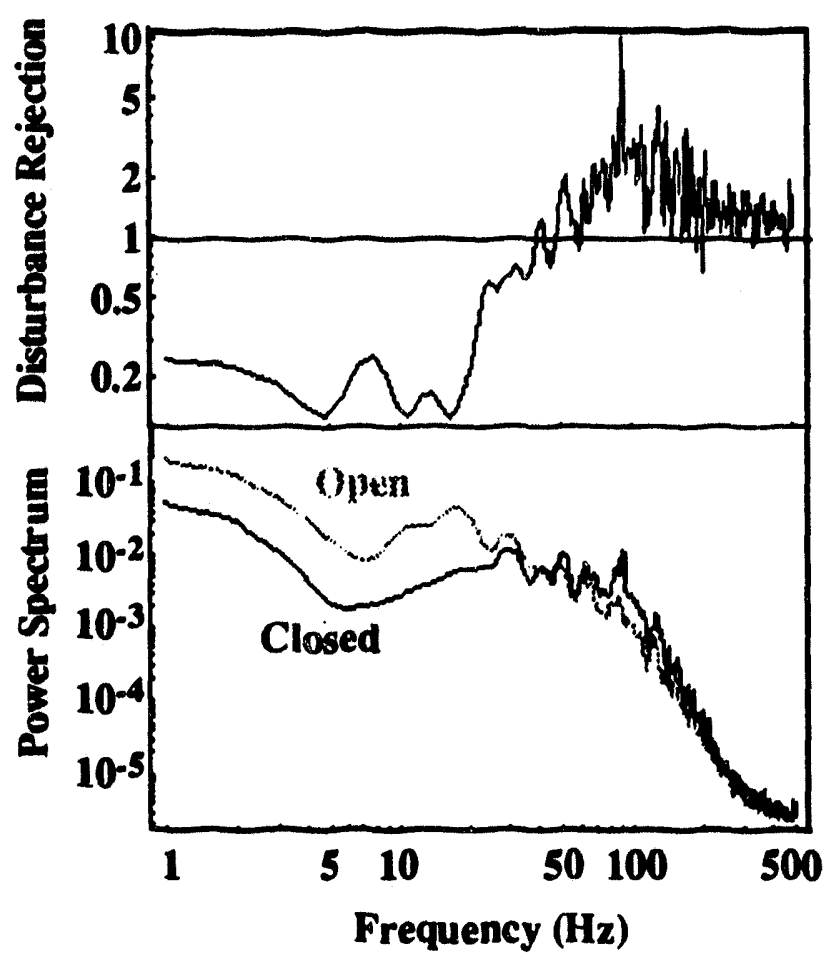

(a)

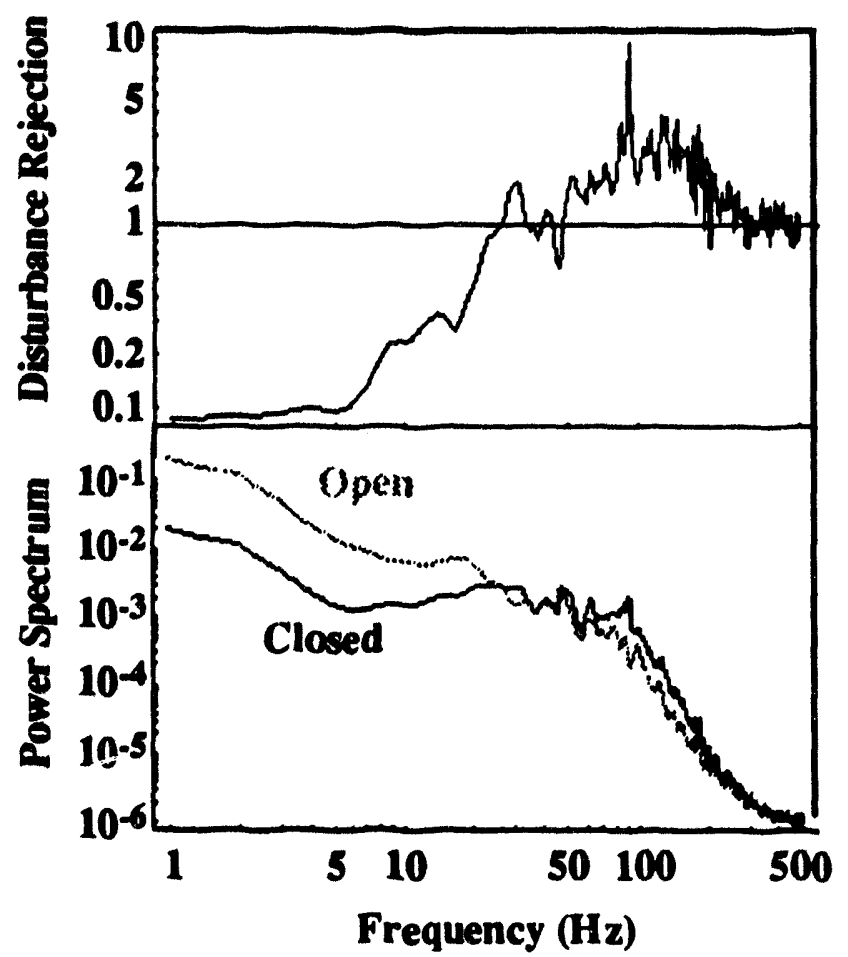

(b)

Fig. 5 Filtered $x$-component (a) and y-component (b) of the power spectrum of the slopes measured by the Hartmann sensor for both open-loop and closed-loop operation. Also shown is the disturbance rejection ratio for this case.

Figure 6 shows the average of 50 captured images for (a) the uncorrected light, (b) only the tilt loop closed, and (c) both the tilt loop and the adaptive optics loop closed. The focus spot for the case where the controller is open has a width of 20 pixels, and the spot appears to be bimodal. Closing the tip/tilt loop appears to have no effect, which suggests that the aberrator appears to induce minimal tilt onto the beam. Closing the adaptive optics loop reduces the width of the spot to 9 pixels, which is about 3 times the diffraction limit. The peak intensity increases by about a factor of 3 . Single frames at $1 / 30$ th second exposures show litule improvement over the average of $\mathbf{5 0}$ frames.

The rms wavefront error measured by the Hartmann sensor, both open loop and closed loop, over a 1 second interval with 1 msec between samples is shown in Fig. 7. The wavefront error was integrated from the gradient data by finite differences and a least-squares method. The finite difference formulas used were taken from Salmon et al. ${ }^{8}$ Closing the adaptive optics loop and tip/tilt loop improved the average $\mathrm{ms}$ wavefront error from 0.38 waves to 0.17 waves ( $56 \%$ reduction). Note that the amplitude of the higher frequencies appears to be higher when the loops are closed, which is confirmed by the large net gain at frequencies above the unity gain crossover frequency shown in Fig. 5. 

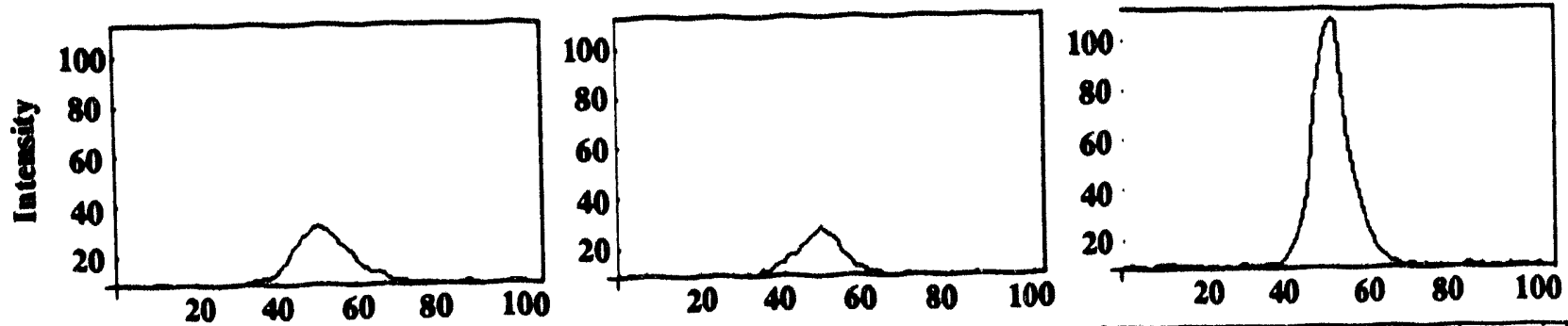

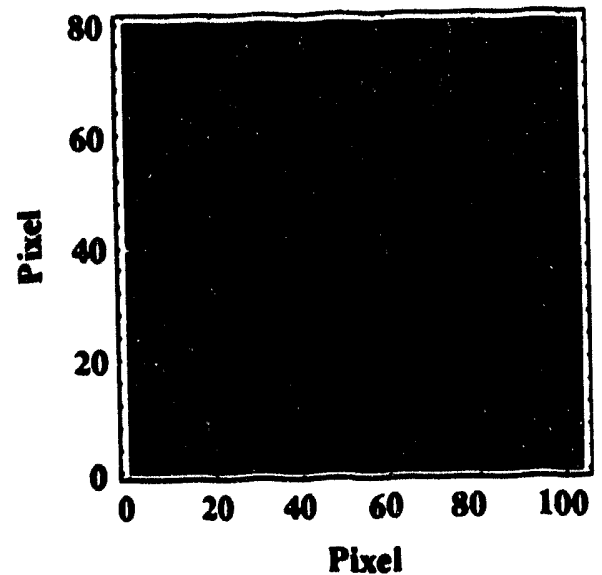

(a)

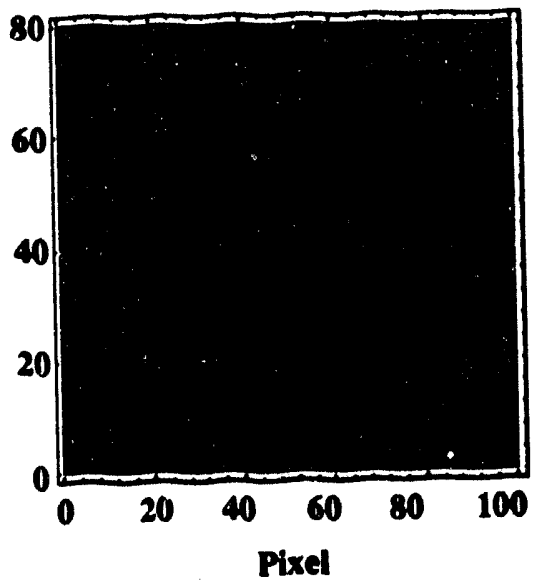

(b)

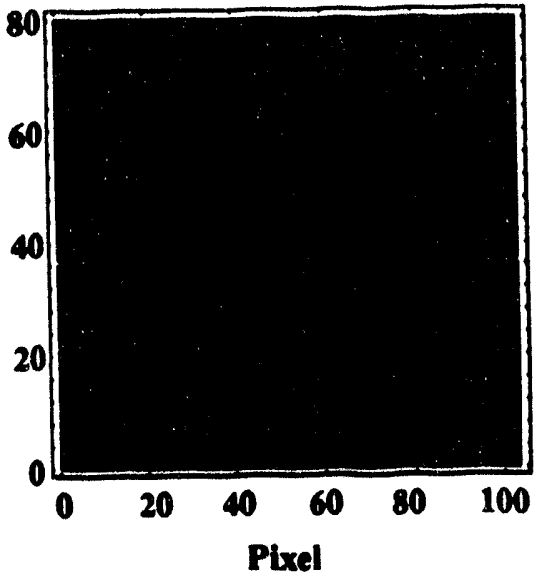

(c)

Fig. 6 Images and corresponding intensity distributions in the science camera for (a) the tip/tilt loop and the adaptive optics loop both open, (b) the tip/tilt loop closed and the adaptive optics loop open, and (c) the tip/tilt loop and the adaptive optics loop both closed. White horizontal lines across the images indicate location of intensity distribution shown above.

The Strehl ratios shown in Fig. 8 are calculated from the absolute reconstructed wavefront errors used in the ms wavefront calculations shown in Fig. 7. The Strehl ratios were calculated by evaluating the Fourier Transform of the wavefront error distribution at the origin of the Fourier Transform plane and comparing that to the same intensity distribution having a flat wavefront, which results in the Strehl ratio equal to

$$
\frac{1}{N^{2}}\left|\sum_{j=1}^{N} e^{-i 2 \pi \phi_{j}}\right|^{2}
$$

where $\mathbf{N}$ is the number of samples in the wavefront, Tor distribution obtained from each finite difference calculation and $\phi$ is the wavefront error distribution in wavelengths of lignt. Note that when $\phi_{j}$ is constant for all $j$, the above ratio reduces to 1 .

When the control loops were opet: : average Strehl ratio was 0.06 . Closing the control loops increased the Strehl ratio by about a factor of 6 to 0.36, which is bout twice the relative increase measured and shown in Fig. 6. Much of this discrepancy is probably caused by the spatial frequency content of the mylar sheet used as the aberration source. Since the aberration is a static source that is swept across the field of view of the adaptive optics package, there is a definite relationship between the temporal frequencies measured and shown in Fig. 5 and the spatial frequencies of the aberrations. The measured amplitude of the disturbance drops as a function of temporal frequencies at a rate that is the square root of the rate predicted for Kolmogorov turbulence. Since the spatial frequencies would also exhibit a similar behavior, the mylar sheet probably has sizable aberrations at scales that are both undetectable with the Hartmann sensor used by the adaptive optics controller and uncorrecwole by the adaptive optics system. These aberrations, however, do affect the size of the focus spot on the imaging detector. Future activities will include finding an aberration source that more closely approximates the atmosphere. 


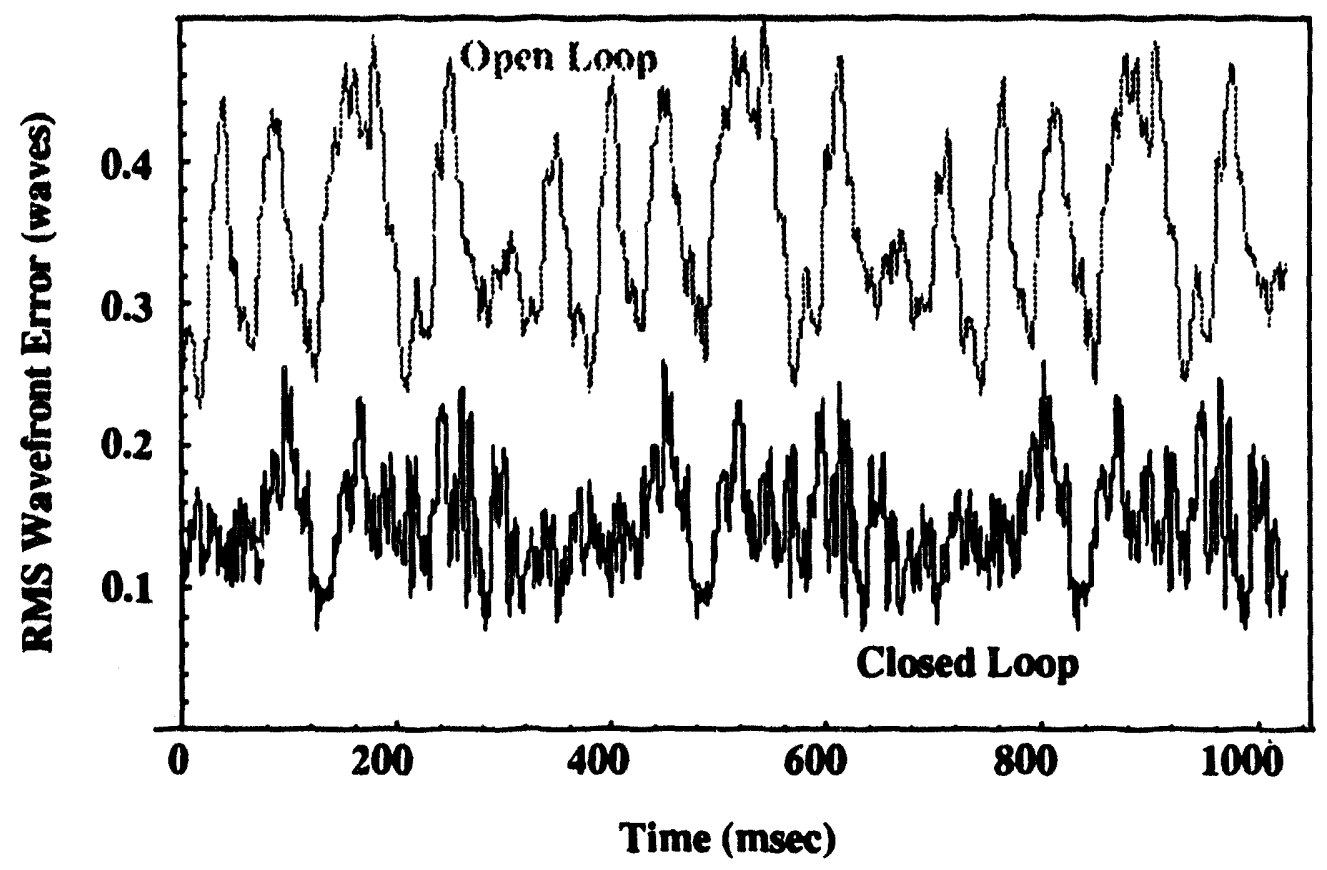

Fig. 7 RMS wavefront error measured by the Hartmann sensor for both open loop and closed loop operation. Average rms error is reduced by about $60 \%$ by adaptive optics controller.

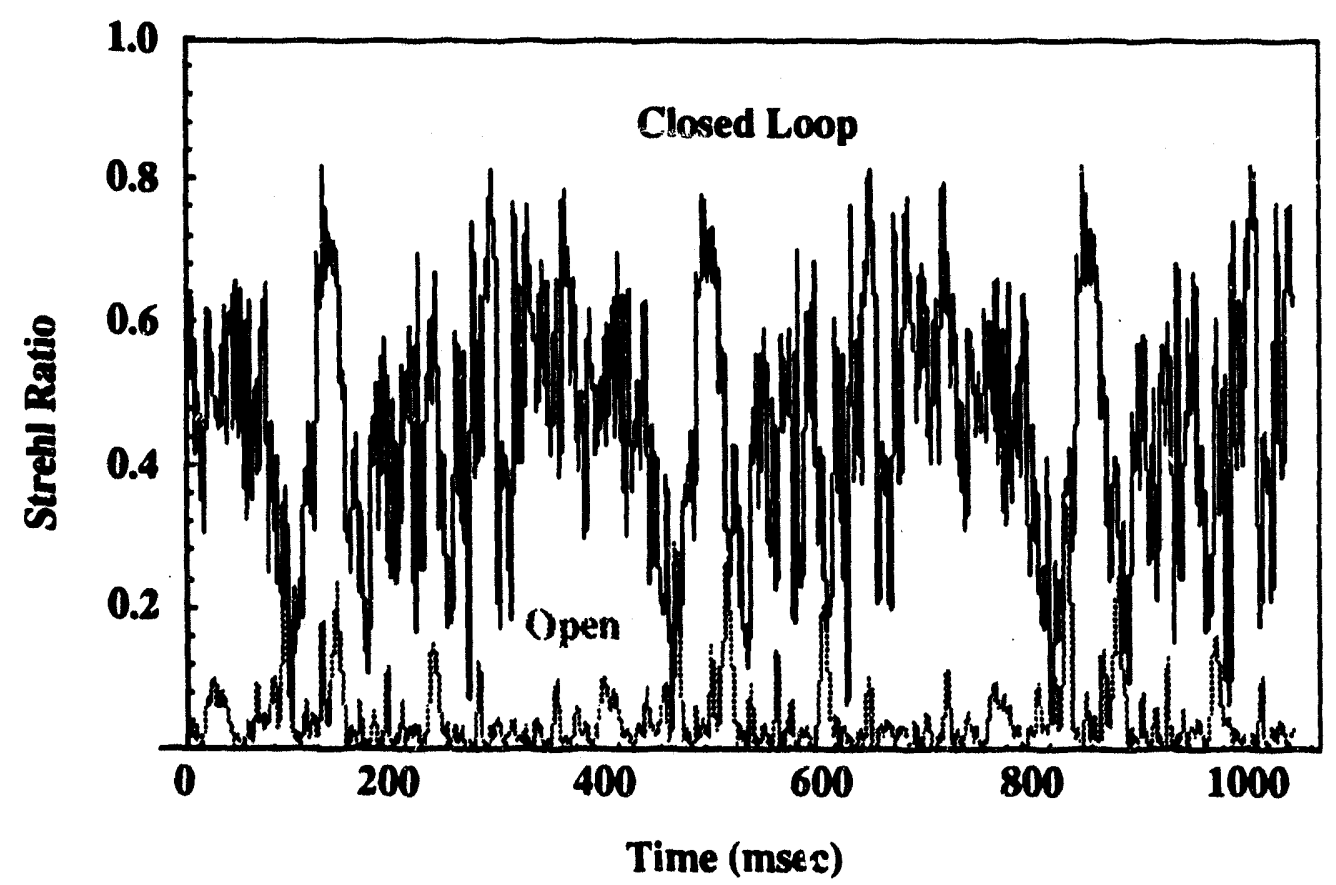

Fig. 8 Strehl ratio calculated from the residual ms data for both open loop and closed loop operation. These calculations do not include degradation in the Strehl ratio from tilt errors. 


\section{CONCLUSION}

A very compact adaptive opucs package has been designed, assembled, and tested in the laboratory. This package is appropriate for meter class telescopes at a good astronomical site and locates thermal sources to the outside of the package for easy isolation from the optical path. The package has successfully closed a control loop in a laboratory on a simulated sodium source and viewed through a simulated aberration source. The unity gain crossover of the controller is $30 \mathrm{~Hz}$ with a peak overshoot gain of 3 . The Strehl ratio was increased by a factor of 3 by closing both control loops. Future activities include improving the controiler and finding a suitable aberration source. Atmospheric experiments with a laser guide star depend on the reactivation of the AVLIS laser in the near future.

\section{ACKNOWLEDGMENTS}

This research was performed under the auspices of the U. S. Department of Energy, under contract number W-7405-ENG-48 to the Lawrence Livermore National Laboratory. The LLNL Laser Guide Star Project is supported by the LLNL Laboratory Directed Research and Development Program. Related work is supported by the Institute of Geophysics and Planetary Physics and by the Engineering Department at LLNL.

\section{REFERENCES}

1. W. Happer, G. MacDonald, C. Max, and F. Dyson, "Atmospheric turbulence compensation using resonant backscautering from the sodium layer in the upper atmosphere," J. Opt. Soc. Am. A. 11, 263 (1994).

2. R. Foy and A. Labeyrie, "Feasibility of adaptive optics telescopes with laser probe," Astronomy and Astrophysics 152, L29 (1985).

3. L. A. Thompson and C. S. Gardner, "Experiments on laser guide stars at Mauna Kea Observatory for adaptive imaging in astronomy," Nature 328, 229 (1987).

4. R. A. Humphries, C. Primmerman, L. Bradley, and J. Hermann, "Atmospheric turbulence measurements using a synthetic beacon in the mesospheric sodium layer," Optics Letters 16, 1367 (1991).

5. R. Q. Fugate, B. L. Ellerbroek, C. H. Higgins, M. P. Jelonek, W. J. Lange, A. C. Slavin, W. J. Wild, J. M. Spinhirne, B. R. Boeke, R. E. Ruane, J. F. Moroney, M. D. Oliker, D. W. Swindle, and R. A. Cleis, "Two generations of laser-guide-star adaptive-optics experiments at the Starfire Optical Range," J. Opt. Soc. Am. A. 11, 310 (1994).

6. R. P. Hackel and B. E. Warner, "The copper-pumped dye laser system at Lawrence Livermore National Laboratory," Proc. SPIE 1859, 120 (1993).

7. K. Avicola, J. Brase, J. Morris, H. Bissinger, H. Friedman, D. Gavel, R. Kiefer, C. Max, S. Olivier, D. Rapp, T. Salmon, D. Smauley, and K. Waltjen, "Sodium laser guide star system at Lawrence Livermore National Laboratory: system description and experimental results," to be published in Proc SPIE 2201, No. 2201-34 (1994).

8. J. T. Salmon, J. W. Bergum, M. W. Kartz, R. W. Presta, and C. D. Swift, "Wavefront correction system based on an equilateral triangular arrangement of actuators," Proc. SPIE 1920, 20 (1993).

9. D. L. Fried, "Least-square fitting a wave-front distortion estimate to an array of phase-difference measurements," $J$. Opt. Soc. Am. 67, 370 (1977).

10. C. D. Swift, J. W. Bergum, J. T. Salmon, J. M. Swan, and C. L. Weinzapfel, "A hexagonal deformable mirror design for AVLIS and Astronomy," in preparation for publication.

11. J. S. Toeppen, E. S. Bliss, T. W. Long, and J. T. Salmon, "Video Hartmann wavefront diagnostic that incorporates a monolithic microlens array," Proc. SPIE 1544, 218 (1991).

12. J. M. Brase, K. Avicola, H. D. Bissinger, H. W. Friedman, D. T. Gavel, B. Johnston, C. E. Max, S. S. Olivier, R. W. Presta, D. A. Rapp, J. T. Salmon, and K. E. Waltjen, and W. A. Fisher, "Adaptive optics at Lick Observatory: system architecture and operations," to be published in Proc. SPIE 2201, (1994).

13. R. K. Tyson, Principles of Adaptive Optics, p. 35-6, Academic Press, New York, 1991. 

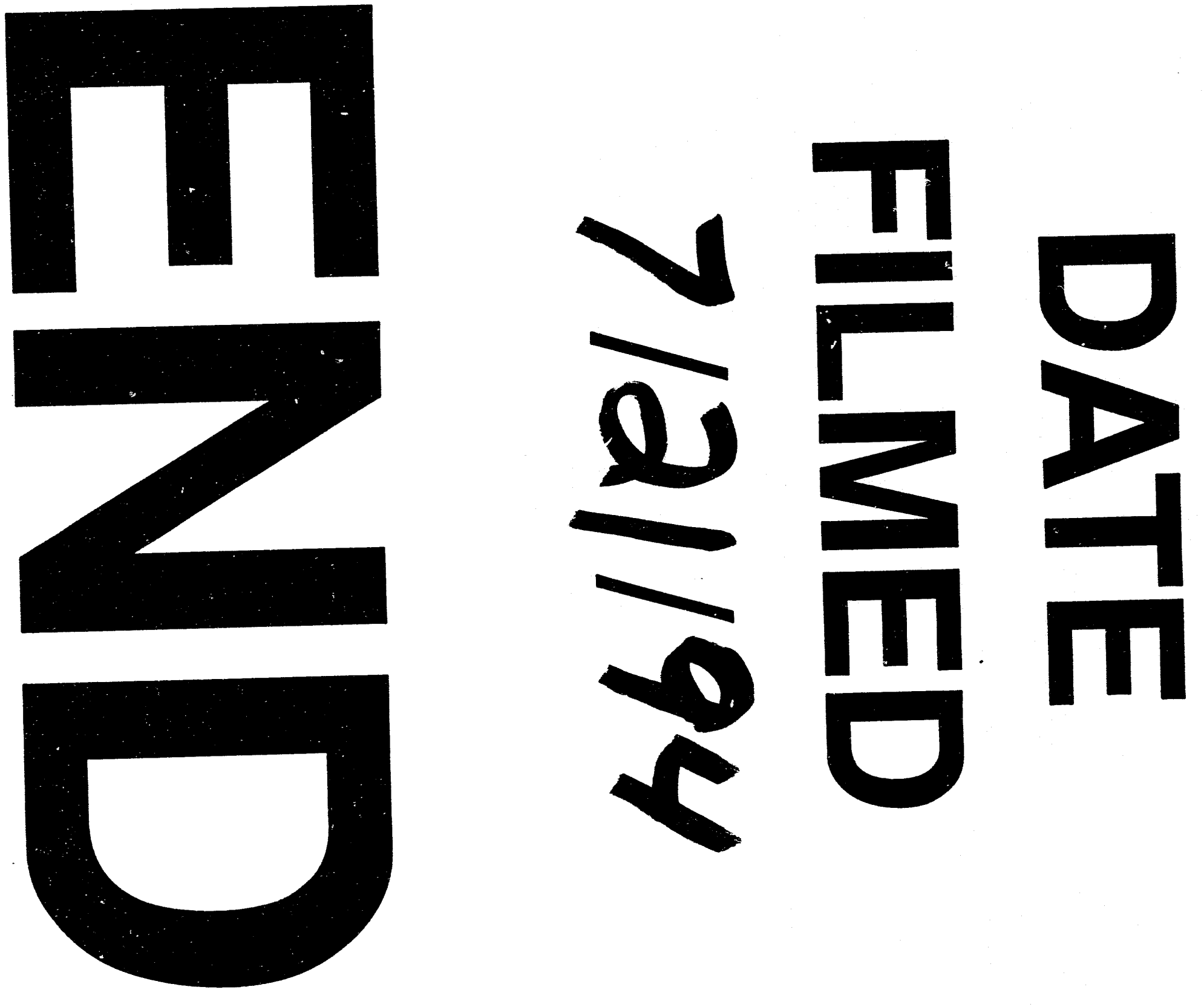


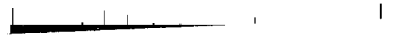$$
\text { , ‘ }
$$ 